\title{
Recent Incidence Trend of Surgically Resected Esophagogastric Junction Adenocarcinoma and Microsatellite Instability Status in Japanese Patients
}

\author{
Yu Imamura, b Masayuki Watanabe ${ }^{a}$ Tasuku Toihata ${ }^{a, b}$ Manabu Takamatsu ${ }^{c}$ \\ Hiroshi Kawachic Ikumi Haraguchi ${ }^{d}$ Yoko Ogatab Naoya Yoshidab Hiroshi Saekie \\ Eiji Oki $^{\text {e Kenichi Taguchi }}{ }^{f}$ Manabu Yamamotog ${ }^{9}$ Masaru Moritag $^{g}$ Shinji Mine ${ }^{a}$ \\ Naoki Hikia Hideo Babab Takeshi Sano ${ }^{a}$ \\ ${ }^{a}$ Department of Gastroenterological Surgery, Cancer Institute Hospital of Japanese Foundation of Cancer Research, \\ Tokyo, Japan; bepartment of Gastroenterological Surgery, Graduate School of Medical Sciences, Kumamoto University, \\ Kumamoto, Japan; 'Department of Pathology, Cancer Institute Hospital of Japanese Foundation of Cancer Research, \\ Tokyo, Japan; 'Cancer Precision Medicine Center, Cancer Institute of Japanese Foundation of Cancer Research, Tokyo, \\ Japan; e Department of Surgery and Science, Graduate School of Medical Sciences, Kyushu University, Fukuoka, Japan; \\ fDepartment of Pathology, National Hospital Organization, Kyushu Cancer Center, Fukuoka, Japan; ${ }^{9}$ Department of \\ Gastroenterological Surgery, National Hospital Organization, Kyushu Cancer Center, Fukuoka, Japan
}

\section{Keywords}

Esophagogastric junction · Barrett's esophagus ·

Esophageal adenocarcinoma · Microsatellite instability ·

Molecular subtype

\begin{abstract}
Background: The incidence trend of esophagogastric junction (EGJ) adenocarcinoma in Japan has not been sufficiently investigated. Little is known about the microsatellite instability (MSI) status of this tumor. Summary: Previously published studies analyzing the trend of EGJ adenocarcinoma in Japan were reviewed. And a trend of surgically resected cases (Siewert type I-III) utilizing a retrospective multicenter cohort of 379 patients from 4 academic institutions in Japan investigated. Although an increasing trend in the last 2 reports was considered controversial, our cohort demonstrated a growing number of EGJ adenocarcinoma cases between 2006 and 2013. This trend was evident, especially in
\end{abstract}

Siewert type I cases. In the previous 16 studies that performed MSI testing, MSI-high tumors ranged 0-8.3\%, though there were no fixed microsatellite markers on EGJ adenocarcinoma. In a recent comprehensive genetic analysis by The Cancer Genome Atlas, MSI testing using the following 7 markers, BAT25, BAT26, BAT40, D2S123, D5S346, D17S250 and TGFR-II showed a favorable correlation with hypermutated tumors. We performed MSI testing using 6 of those markers, except TGFR-II, on 206 cases from one institution, and detected 15 cases (7.3\%) with MSI-high. The prevalence of MSI-high was $0 \%$ in Siewert type I, $7.6 \%$ in type II, and $16.7 \%$ in type III. Key message: The number of surgically resected EGJ adenocarcinoma cases gradually increased, and MSI-high was infrequent in Siewert type I-II tumors in our Japanese cohort. Considering MSI-high as a predictive biomarker for emerging immune checkpoint inhibitors, MSI status is becoming more beneficial in EGJ adenocarcinoma.

(c) 2018 S. Karger AG, Basel

\section{KARGER}

(c) 2018 S. Karger AG, Basel

E-Mail karger@karger.com

www.karger.com/dig
Yu Imamura, MD, PhD, FACS

Department of Gastroenterological Surgery

Cancer Institute Hospital of Japanese Foundation of Cancer Research

3-8-31, Ariake, Koto-ku, Tokyo 135-8550 (Japan)

E-Mail yu.imamura@jfcr.or.jp 


\section{Introduction}

In Western countries, the incidence of esophagogastric junction (EGJ) adenocarcinoma has been increasing gradually over the past few decades, whereas that of noncardiac gastric cancer decreased with declining prevalence of Helicobacter pylori infection [1]. EGJ adenocarcinoma, also called as esophageal adenocarcinoma, generally develops from a premalignant lesion of esophageal columnar metaplasia of Barrett's esophagus (BE) in the West. Despite the recent advancements in multimodal treatments, this tumor is still an aggressive malignancy with poor outcome.

In a number of clinical trials of conventional chemotherapeutic agents or molecular targeted drugs, patients with EGJ adenocarcinoma often share treatment strategy with gastric adenocarcinoma cases, since these 2 diseases are considered to be similar. Recently, immune checkpoint inhibitor has emerged as a novel effective chemotherapeutic option across a broad spectrum of malignant tumors and gastrointestinal adenocarcinoma. The phase 3 trial ONO-4538-12 (ATTRACTION-2) revealed the efficacy and safety of programmed cell death protein 1 (PD1) blockade by monoclonal antibody nivolumab in Asian patients with esophagogastric $(n=30)$ and gastric adenocarcinoma $(n=272)$ [2]. Currently, in terms of a predictor of response to immune checkpoint inhibitor, much focus is being placed on the microsatellite instability (MSI) status [3]. However, little is known about the MSI status in EGJ adenocarcinoma.

In this study, we reviewed the previous studies investigating the recent incidence trend of EGJ adenocarcinoma cases in Japan, and also examined its trend utilizing 379 surgically resected Siewert type I, II, and III EGJ adenocarcinoma cases (type I, tumor epicenter located within 1-5 $\mathrm{cm}$ above the anatomical EGJ; type II, within $1 \mathrm{~cm}$ above and $2 \mathrm{~cm}$ below the EGJ; type III, between 2 and 5 $\mathrm{cm}$ below the EGJ with esophageal infiltration) from our multicenter retrospective cohort study in Japan. In addition, we reviewed previous reports related to the MSI status of this tumor and investigated the MSI status on 206 cases of EGJ adenocarcinoma from one institution.

\section{Recent Trends of EGJ Adenocarcinoma in Japan}

In Japan, Kusano et al. [4] first investigated the incidence trend of Japanese cases of EGJ adenocarcinoma, analyzing 520 cases with surgically resected Siewert type I-III tumors in a single institution of National Cancer
Center (Tokyo) in 2008, one of the highest volume cancer centers in Japan. They demonstrated that the proportion of Siewert type II increased between 1962 and 2005, but that of Siewert type I remained around 1\% over 44 years. Recently, Yamashita et al. [5] showed that the number of surgically resected cases of EGJ adenocarcinoma were gradually increased between 2001 and 2010, using a questionnaire-based national retrospective study in Japan. In their article, EGJ adenocarcinoma was defined as tumors with epicenter located within $2 \mathrm{~cm}$ proximal or distal to the anatomical EGJ by the Japanese classification of gastric cancer (14th edition, Japanese only).

To be aware of current incidence trends of EGJ adenocarcinoma, we retrospectively collected a total of 379 patients who underwent surgical resection between 2006 and 2013 in 4 academic institutions (Department of Gastroenterological Surgery, the Cancer Institute Hospital [Tokyo]; Department of Gastroenterological Surgery, Kumamoto University Hospital [Kumamoto], Department of Surgery and Science, Kyushu University Hospital [Fukuoka], and Department of Surgery, National Hospital Organization Kyushu Cancer Center [Fukuoka]) in Japan. Ethical approvals for this retrospective observational study were obtained from all institutions (approval number 2015-1107 for The Cancer Institute Hospital, 858 for Kumamoto University Hospital, 747-00 for Kyushu University Hospital, and 2015-26 for National Hospital Organization Kyushu Cancer Center). The annual numbers of surgically resected EGJ adenocarcinoma cases were counted according to the tumor locations by Siewert classification. During the 8-year study period, the annual number of surgically resected cases increased gradually (Fig. 1a). Of note, the increase in Siewert type I tumors was evident. While that of Siewert type III tumors decreased (Fig. 1a). These tendencies were clearer in terms of proportions by Siewert classification (Fig. 1b). Although the report from Yamashita et al. [5] and our data showed growing trends of EGJ adenocarcinoma, these were attributed to an increase of the elderly population in Japan. The search in PubMed revealed that there were no reports examining the incidence trend of EGJ adenocarcinoma in other Asian countries, such as Korea or China. Further populationbased study is warranted to clarify the trend of this tumor.

Anatomically, EGJ adenocarcinoma includes tumors arising from $\mathrm{BE}$, and those arising from cardiac mucosa. Theoretically, tumors adjacent to BE can presumably originate from the $\mathrm{BE}$ (BE-associated EGJ adenocarcino$\mathrm{ma})$. We also investigated the annual number of surgically resected cases with EGJ adenocarcinoma, according to the presence or absence of BE in our cohort $(n=379)$. 


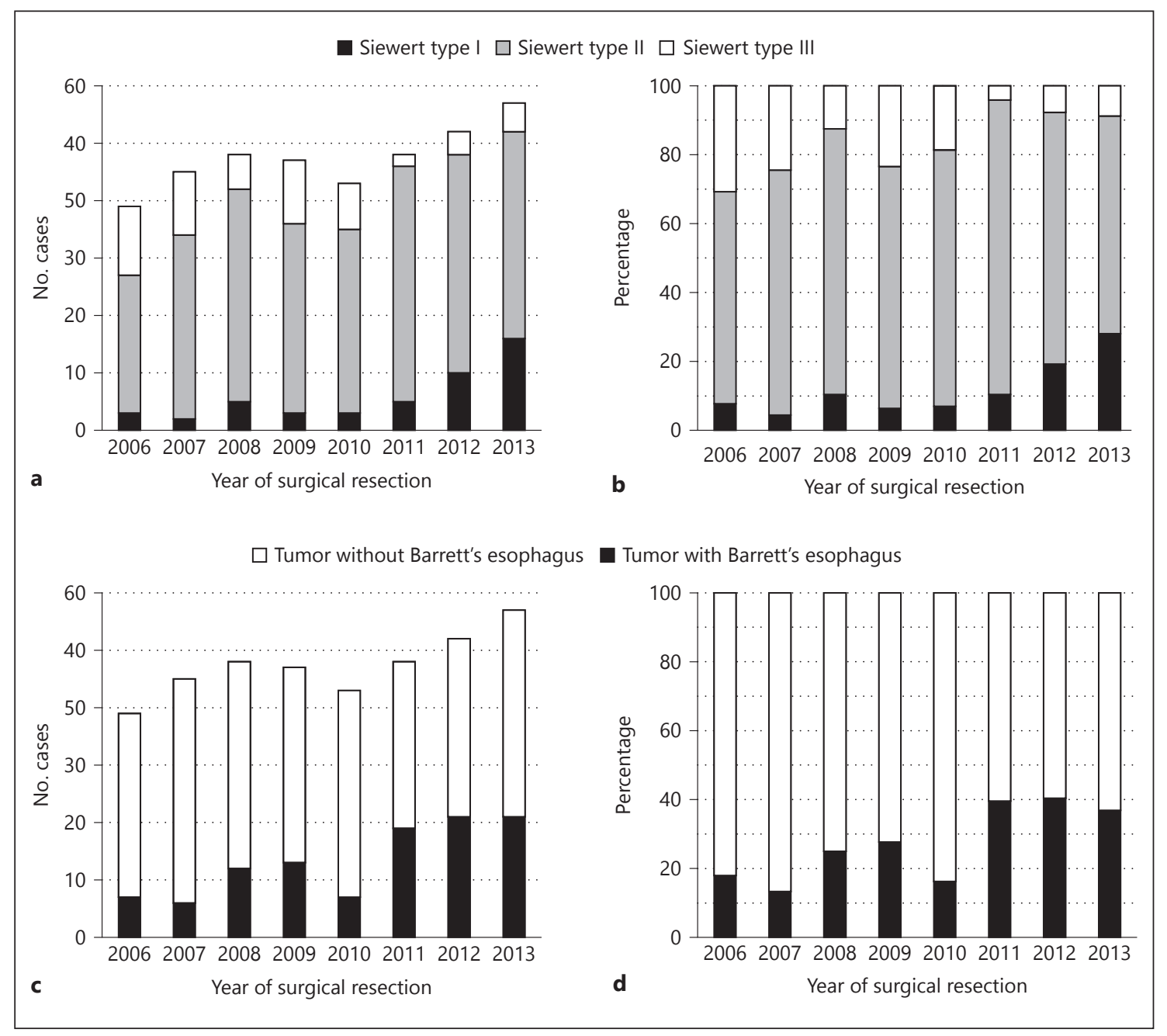

Fig. 1. Recent trends of surgically resected cases of esophagogastric junction adenocarcinoma in 4 institutions in Japan $(n=379)$. $\mathbf{a}$ and $\mathbf{b}$, patient numbers (a) and proportions (b) according to tu-

The existence of BE was defined by endoscopic findings, or by surgical pathology reports. The proportions of tumors coexisting with BE were 35/47 (74.5\%). Siewert type I tumors, 69/273 (25.3\%) in Siewert type II, and 2/59 $(3.4 \%)$ in Siewert type III. Type I tumors were significantly associated with coexistence of BE $(p<0.0001)$. Over the 8 years, we observed a growing trend of tumors adjacent to $\mathrm{BE}$, in terms of numbers and ratios (Fig. 1c, d). Our data may suggest that BE-associated EGJ adenocarcinoma cases are increasing in Japan, similar to what was observed in Western countries a few decades ago. However, as our cohort were collected in a retrospective manner, a future larger scale prospective study in collaboration with endoscopists and pathologists would be needed to address the trend of BE-associated tumors. mor location by Siewert classification. $\mathbf{c}$ and $\mathbf{d}$, patient numbers (c) and proportions (d) according to the presence or absence of Barrett's esophagus (BE).

\section{MSI and Gastrointestinal Cancers}

Genetic instability is regarded as a hallmark of malignant tumor. MSI is a form of genetic instability in cancer, caused by DNA mismatch repair deficiency (MMR) [6]. Microsatellites are short tandem repeats of simple DNA sequence, generally 5-60 times of mono-, di-, tetra- penta- or hexanucleotide throughout the genome. Around 50,000-100,000 microsatellites occur in human genome, commonly in introns, promoter regions, as well as in exons. DNA synthesis is always at risk of replicative error of DNA repairing or replication. This nature of microsatellites results in a susceptibility to replication errors caused by slippage of DNA polymerase over short tandem repeats. In normal cells, these replicative errors can be ef- 
ficiently corrected by MMR proteins, such as MLH1, MSH2, MSH6, and PMS2 [7]. The MMR deficiency, usually caused by genetic or epigenetic alterations, is one of the major genetic predispositions to cancer.

High-level MSI (MSI-high) was approved as a biomarker for pembrolizumab in many types of solid malignancies by Food and Drug Administration (FDA). In general, MSI-high tumors possess remarkably high mutational burden, potentially producing a numerous number of neoantigens [8]. Displaying these tumor-specific neoantigen by antigen-presenting cells recruit $\mathrm{CD} 8+\mathrm{T}$ cells (effector T cells) into tumor microenvironment. Simultaneously, tumor cells promote immune evasion by suppressing T-cell mediated tumor cell lysis, through programmed death ligand 1 (PD-L1) [9]. Immune check point inhibitors, such as nivolumab and pembrolizumab, restore $\mathrm{T}$-cell activation by blocking the interaction between PD-L1 on tumor cells and PD-1 on activated T cells, leading to antitumor response. This is why MSI status can currently be used as a predictor of PD-1 blockade in gastric and colorectal cancers in gastrointestinal malignancies in the US.

MMR deficiency can be detected by genetic testing or immunohistochemistry (IHC). Genetic testing has been employed for screening hereditary non-polyposis colorectal cancer, which are caused by germline mutations of MMR genes, by the Bethesda panel (BAT25, BAT26, D2123, D5S346, and D17S250) in clinical practice [10]. Although mononucleotide markers (BAT25 and BAT26) are stable and easy to realize when shifted in a fragment analysis, amplicons of dinucleotide markers (D2123, D5S346, and D17S250) are less sensitive due to the effect of loss of heterozygosity. In recent times, Promega MSI Analysis System (ver.1.2, Madison, WI, USA) using 5 mononucleotide markers alone (BAT25, BAT26, MONO27, NR21, and NR24) are becoming more common.

The reproducibility of MSI genetic testing has already been verified to be quite high across 71 clinical laboratories [11]. Genetic testing allows visualization of amplified fragments of MSI markers, resulting in a better agreement. On the contrary, the sensitivity of IHC methods always depends on the antibodies used, as well as on the quality of formalin-fixed paraffin-embedded (FFPE) tissue. It is sometimes difficult to judge whether uneven staining is caused by loss of expression, or simply due to degraded protein on FFPE tissue. In addition, when a mutation occurs outside MLH1-PMS2 interacting domain, both MLH1 and PMS2 expressions can be detected theoretically. Because IHC method sometimes miss MMR de- ficiency in some situations, genetic testing would be recommended rather than IHC methods, for detecting MMR deficiency.

\section{Previous Studies Examining MSI Status in EGJ Adenocarcinoma}

There is still no consensus on which microsatellite markers should be used for detecting MSI-high in EGJ adenocarcinoma. In colorectal adenocarcinoma, the Bethesda panel mentioned above, was developed for detecting hereditary non-polyposis colorectal cancer at $\mathrm{Na}$ tional Cancer Institute-sponsored MSI workshop in 1997 [10]. To our best knowledge, there are 16 studies that investigated the MSI status, focusing on EGJ adenocarcinoma (Table 1) [12-27]. All those studies used genetic testing. Among them, 13 studies (81.3\%) employed at least 1 microsatellite marker listed in the Bethesda panel. In a recent comprehensive genomic study of esophageal cancer by The Cancer Genome Atlas (TCGA) Research Network, microsatellite markers of BAT40 and TGFR-II were added to the Bethesda panel, and demonstrated that 7 cases $(7 / 172,4.1 \%$ of esophageal adenocarcinoma) with MSI-high tumors were hypermutated in their data set, harboring 32-103 mutations occurred per mega base [17]. Hence, the following 7 microsatellite markers, BAT25, BAT26, BAT40, D2S123, D5S346, D17S250 and TGFR-II, are suitable for detecting MSI-high tumors in EGJ adenocarcinoma.

Among the 16 studies, 8 studies investigated the prevalence of MSI-high tumors (Table 1). Although the definitions of MSI-high tumors were not common across the studies, all studies marked low prevalence of MSI-high in EGJ adenocarcinoma, ranging from 0 to $8.3 \%$ (Table 1 ). The number of patients analyzed was very small $(n \leq 80)$, and the statistical power was limited, except 1 study from TCGA ( $n=172$, data were obtained from supplementary data) [17]. There were only a few studies investigating the association between MSI-high and other molecular status, or clinicopathological characteristics in EGJ adenocarcinoma.

Kulke et al. [19] did not detect any MSI-high tumor (defined as 3 or more unstable microsatellite markers of 5 markers) in their cohort $(n=70)$. They suggested a better prognosis of MSI-low tumors $(n=13$, defined as 1 or 2 unstable microsatellite markers), compared to MSI-absent cases $(n=57)$, but it did not reach a statistical significance $(p=0.12)$ [19]. They did not find any outstanding clinicopathological features of MSI-low. 
Table 1. Previous 16 studies examining microsatellite instability (MSI) in esophagogastric adenocarcinoma

\begin{tabular}{llll}
\hline Ref. First & Year of \\
No. author & publication & $\begin{array}{l}\text { No. } \\
\text { cases }\end{array}$ & $\begin{array}{l}\text { No. } \\
\text { MSI-high, } \\
n(\%)\end{array}$
\end{tabular}

12 Shiraishi $2006 \quad 12 \quad 1(8.3) \quad 30 \%$ unstable markers NA

BAT25, BAT26, D2S123, D5S346, D17S250, D17S579, D17S786, D17S796, D3S1300, D7S486, D9S161, D11S904, D13S268, D18S474

\begin{tabular}{llllll}
\hline 3 & Pandilla 2013 & $2(6.7)$ & $\geq 2$ unstable markers & NA & $\begin{array}{l}\text { D2S123, D3S1300, D5S346, } \\
\text { D9S1748, D17S250, TP53CA }\end{array}$
\end{tabular}

14 Farris $2011 \quad 76 \quad 5(6.6) \quad \geq 2$ unstable markers $\quad$ BE-associated $\quad \begin{aligned} & \text { BAT25, BAT26, D2S123, D5S346, } \\ & \text { D17S250 }\end{aligned}$

15 Chong $2013 \quad 54 \quad 3(5.6) \quad \geq 2$ unstable markers $\quad$ Siewert type II/III

BAT25, BAT26, NR21, NR24,

\begin{tabular}{llllll}
\hline 16 & Dolan 2004 & $2(4.3) \quad 2$ unstable markers & Distal esophagus & D3S587, D5S107, D5S346, D9S171, \\
D17S805, D18S70
\end{tabular}

\begin{tabular}{lllllll}
\hline 17 & TCGA & 2017 & 172 & $7(4.1)$ & $\geq 40 \%$ unstable markers NA \\
\hline 18 & Evans & 2004 & 27 & 0 & $\geq 5$ unstable markers & NA
\end{tabular}

BAT25, BAT26, BAT40, TGFR-II, D2S123, D5S346, D17S250

D2S123, D10S197, D2S119, D11S904, D2S147, D3S1764, D7S1830, D7S1805, D2S434, D9S299, BAT25, BAT26, D5S346, D17S250, TGF- $\beta$-RII

\begin{tabular}{llllll}
\hline Kulke $2001 \quad 80$ & 203 unstable markers & BE-associated & $\begin{array}{l}\text { BAT25, BAT26, D10S219, D10S541, } \\
\text { D10S551 }\end{array}$ \\
\hline
\end{tabular}

\begin{tabular}{llllll}
\hline 20 & Shirasaki 2009 & 11 & NA & NA & NA
\end{tabular}

\section{BAT25, BAT26, D2S123, D5S346,} D17S250, D17S796, TP53, D17S579, D17S786, D3S1300, D7S486, D9S161, D11S904, D13S268, D18S474

\begin{tabular}{lllllll}
\hline 21 & Cai & 2008 & 18 & NA & NA & Distal esophagus \\
\hline 22 & Lin & 2006 & 44 & NA & NA & NA
\end{tabular}

D2S123, D3S1300, D3S1616, D5S346, D17S787, D18S58, BATRII D3S1754, D2S119, BAT26, D2S123, BAT21, D3S3603, AFM072yb7, D3S2314, D3S3609, CI_4_1, D10S2314, D10S2318, D10S197, D10S541, D10S2309, D10S2311, D10S2317, D10S2312, D10S2310, D11S904, CTG-B37, D13S175, D14S72, D14S69, D17S1157, D17S250, D17S1787, D17S856, D21S411, DYS19

\begin{tabular}{|c|c|c|c|c|c|c|c|}
\hline 23 & Yanagi & 2000 & 54 & NA & NA & Siewert type I/III & $\begin{array}{l}\text { D5S346, D18S34, TP53, D14S65, } \\
\text { D14S75, D14S80, D14S267 }\end{array}$ \\
\hline 24 & Rumpel & 1999 & 28 & NA & NA & NA & $\begin{array}{l}\text { D4S392, D4S1543, D4S2915, } \\
\text { D4S2932, D4S1538, D4S1534, } \\
\text { D4S423, D4S1638, D4S1572, D4S407, } \\
\text { IL2, D4S427, D4S1615, D4S1565, } \\
\text { D4S1586, D4S1589, D4S620, } \\
\text { D4S3028, D4S1607, D4S2951, } \\
\text { D4S2920, D4S1554, D4S1535, } \\
\text { D4S171, D4S426 }\end{array}$ \\
\hline
\end{tabular}


Table 1 (continued)

\begin{tabular}{|c|c|c|c|c|c|c|c|}
\hline $\begin{array}{l}\text { Ref. } \\
\text { No. }\end{array}$ & $\begin{array}{l}\text { First } \\
\text { author }\end{array}$ & $\begin{array}{l}\text { Year of } \\
\text { publication }\end{array}$ & $\begin{array}{l}\text { No. } \\
\text { cases }\end{array}$ & $\begin{array}{l}\text { No. } \\
\text { MSI-high, } \\
n(\%)\end{array}$ & Definition of MSI-high & Tumor location & Microsatellite markers \\
\hline
\end{tabular}

Microsatellite markers listed of the Bethesda panel (BAT25, BAT26, D2S123, D5S346, and D17S250) are bold.

BE, Barrett's esophagus; NA, not applicable; TCGA, The Cancer Genome Atlas.

Farris et al. [14] demonstrated that patients with MSIhigh tumors were significantly associated with advanced age (76.2 \pm 5.9 years old in MSI-high [ $n=5], 66.4 \pm 11.0$ years old in MSI-absent [ $n=71$ ], $p=0.006)$, and higher number of tumor infiltrating lymphocytes $(42.9 \pm 5.9$ per high power field in MSI-high, $5.2 \pm 13.0$ per high power field in MSI-absent, $p<0.0001$ ). They also addressed the pathological characteristics according to MSI status. Generally, MSI-high tumors are associated with medullary morphology in gastric and colorectal adenocarcinoma, suggesting similar histological characteristics across gastrointestinal adenocarcinoma. Expectedly, in their observation, 2 of 5 (40\%) cases with MSI-high tumor exhibited medullary component. The remaining 3 cases (60\%) included one with conventional adenocarcinoma without any additional component, another with signet-ring cell carcinoma, and the other with mucinous histology. Signet-ring cell and mucinous component are reportedly related to CpG island methylator phenotype, as well as MSI-high in colorectal adenocarcinoma [28]. MSI-high can be also induced by epigenetic silencing of $\mathrm{MLH1}$ in colorectal adenocarcinoma. Further study would be needed to address the relationship between signet-ring cell or mucinous histology, epigenetic alterations, and MSI status in EGJ adenocarcinoma.

MSI Status in Japanese EGJ

Adenocarcinoma

\section{Prevalence of MSI-high Tumors in Cancer Institute Hospital Cases}

Using FFPE-derived DNA from 206 cases with EGJ adenocarcinoma who underwent surgical resection at Cancer Institute Hospital, MSI testing was performed by fragment analysis. After microdissection of the tumor area marked by pathologists (Y.I. and M.T.) under a microscope from hematoxylin and eosin stained tissue section, DNA was extracted using QIAamp DNA FFPE Tissue Kit (QIAGEN, Duesseldorf, Germany) [29]. DNA fragment analyses were done by ABI PRISM 3030xl Genetic Analyzer (Applied Biosystems, Foster City, CA, USA), using 6 microsatellite markers (BAT25, BAT26, BAT40, D2S123, D5S346, and D17S250; primer sequences conducted are shown in Table 2), as previously described [30]. All these 6 markers overlap the 7 markers used in the TCGA esophageal cancer study. This study was approved by the research institutional review board at Cancer Institute Hospital (approval number 20151107). MSI-high was defined as 2 or more unstable markers, MSI-low as one unstable marker, and microsatellite stable as no unstable marker. MSI-high, MSI-low, and microsatellite stable were detected in $15(7.3 \%), 19(9.2 \%)$, and 172 cases (83.5\%), respectively. Notably, MSI-high was not detected in Siewert type I tumors. As the tumor 
Table 2. Primer sequences for MSI assay in this study

\begin{tabular}{|c|c|c|c|c|}
\hline Markers & Repeating unit & Forward/reverse & $5^{\prime}$ Reporter dye & $5^{\prime}-3^{\prime}$ sequence \\
\hline \multirow[t]{2}{*}{ BAT25 } & \multirow[t]{2}{*}{ Mononucleotide } & Forward & 6-FAM & TCGCCTCCAAGAATGTAAGT \\
\hline & & Reverse & None & TATGGCTCTAAAATGCTCTGTTCTC \\
\hline \multirow[t]{2}{*}{ BAT26 } & \multirow[t]{2}{*}{ Mononucleotide } & Forward & 6-FAM & GAAATTGGATATTGCAGCAGTCAGAG \\
\hline & & Reverse & None & САTTTTTTAАССАТTСААСАТTTTTAАССС \\
\hline BAT40 & Mononucleotide & Forward & 6-FAM & AGCCAAGATTAACTTCCTACACCACAAC \\
\hline D2S123 & Dinucleotide & Reverse & None & GTGTCTTCCTTTCTGACTTGGATACCATCTA \\
\hline \multirow[t]{2}{*}{ D5S346 } & \multirow[t]{2}{*}{ Dinucleotide } & Forward & 6-FAM & ACTCACTCTAGTGATAAATCGGG \\
\hline & & Reverse & None & GTGTCTTAGCAGATAAGACAGTATTACTAGTT \\
\hline \multirow[t]{2}{*}{$\mathrm{D} 17 \mathrm{~S} 250$} & \multirow[t]{2}{*}{ Dinucleotide } & Forward & 6-FAM & TACACATACATAAACTTTCAAATGGTTTC \\
\hline & & Reverse & None & GTGTCTTGCCACTCAGCTGGCCATATATATATT \\
\hline
\end{tabular}

Fig. 2. Proportions of microsatellite instability (MSI) status according to tumor locations by Siewert classification. MSI testing was performed in the patients from Cancer Institute Hospital $(n=206)$. MSS, microsatellite stable.

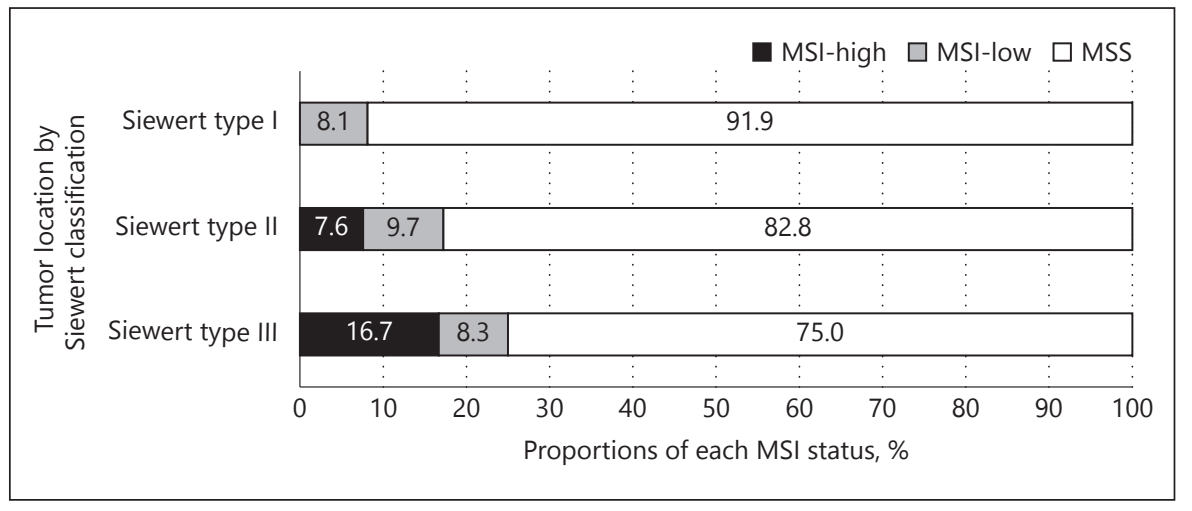

epicenter location go down from the esophagus (Siewert type I) to the stomach (Siewert type III), the proportion of MSI-high tumors increased, and that of MSI-low remained stable (8-9\%, Fig. 2). MSI-high was a relatively rare genetic instability in EGJ adenocarcinoma compared to that in gastric adenocarcinoma, reported to be around $10-25 \%[31,32]$. Further study on the features of MSIlow is warranted.

\section{Conclusion}

Here, we reviewed the incidence trend of surgically resected EGJ adenocarcinoma in Japan, and previous studies regarding the MSI status in this tumor. The number of patients with EGJ adenocarcinoma is on the rise in Japan, similar to the Western countries. Having high expectations of cancer immunotherapy, MSI status is becoming an essential molecular testing in gastrointestinal malignancies including EGJ adenocarcinoma. However, MSI status in EGJ adenocarcinoma has still not been well examined or understood. Further studies examining the clinical, pathological, and molecular characteristics according to MSI status, would be beneficial to improve the treatment outcome of this tumor.

\section{Acknowledgement}

We would like to express our sincere appreciation to Hiroko Taniguchi (Kumamoto University), Yuko Kubota (Kyushu University), and Motoyoshi Iwakoshi (Cancer Institute Hospital) for data acquisition.

\section{Disclosure Statement}

All authors have no conflict of interest to declare.

\section{Grant Support}

This work was supported by JSPS KAKENHI Grant Number JP16H06259 (Y.I.).
12

Digestion 2019;99:6-13

DOI: $10.1159 / 000494406$
Imamura et al. 


\section{References}

1 Blaser MJ: Disappearing Microbiota: Helicobacter pylori protection against esophageal adenocarcinoma. Cancer Prev Res (Phila) 2008;1:308-311.

2 Kang YK, Boku N, Satoh T, et al: Nivolumab in patients with advanced gastric or gastrooesophageal junction cancer refractory to, or intolerant of, at least two previous chemotherapy regimens (Ono-4538-12, Attraction-2): a randomised, double-blind, placebocontrolled, phase 3 trial. Lancet 2017;390: 2461-2471.

3 Gotwals P, Cameron S, Cipolletta D, et al: Prospects for combining targeted and conventional cancer therapy with immunotherapy. Nat Rev Cancer 2017;17:286-301.

4 Kusano C, Gotoda T, Khor CJ, et al: Changing trends in the proportion of adenocarcinoma of the esophagogastric junction in a large tertiary referral center in Japan. J Gastroenterol Hepatol 2008;23:1662-1665.

5 Yamashita H, Seto Y, Sano T, et al: Results of a nation-wide retrospective study of lymphadenectomy for esophagogastric junction carcinoma. Gastric Cancer 2017;20(suppl 1): 69-83.

6 Lengauer C, Kinzler KW, Vogelstein B: Genetic instabilities in human cancers. Nature 1998;396:643-649.

7 Imai K, Yamamoto H: Carcinogenesis and microsatellite instability: the interrelationship between genetics and epigenetics. Carcinogenesis 2008;29:673-680.

8 Yarchoan M, Johnson BA 3rd, Lutz ER, et al: Targeting neoantigens to augment antitumour immunity. Nat Rev Cancer 2017;17: 209-222.

9 Ricklefs FL, Alayo Q, Krenzlin H, et al: Immune evasion mediated by PD-L1 on glioblastoma-derived extracellular vesicles. Sci Adv 2018;4:eaar2766.

10 Boland CR, Thibodeau SN, Hamilton SR, et al: A national cancer institute workshop on microsatellite instability for cancer detection and familial predisposition: development of international criteria for the determination of microsatellite instability in colorectal cancer. Cancer Res 1998;58:5248-5257.

11 Zhang L: Immunohistochemistry versus microsatellite instability testing for screening colorectal cancer patients at risk for hereditary nonpolyposis colorectal cancer syndrome. Part I. The utility of microsatellite instability testing. J Mol Diagn 2008;10:301-307.

12 Shiraishi H, Mikami T, Yoshida T, et al: Early genetic instability of both epithelial and stromal cells in esophageal squamous cell carcinomas, contrasted with Barrett's adenocarcinomas. J Gastroenterol 2006;41:1186-1196.

13 Pandilla R, Kotapalli V, Gowrishankar S, et al: Distinct genetic aberrations in oesophageal adeno and squamous carcinoma. Eur J Clin Invest 2013;43:1233-1239.

14 Farris AB 3rd, Demicco EG, Le LP, et al: Clinicopathologic and molecular profiles of microsatellite unstable Barrett esophagus-associated adenocarcinoma. Am J Surg Pathol 2011; 35:647-655.

15 Chong IY, Cunningham D, Barber LJ, et al: The genomic landscape of oesophagogastric junctional adenocarcinoma. J Pathol 2013; 231:301-310.

16 Dolan K, Morris AI, Gosney JR, et al: Three different subsite classification systems for carcinomas in the proximity of the Gej, but is it all one disease? J Gastroenterol Hepatol 2004; 19:24-30.

17 The Cancer Genome Atlas Research Net work: Integrated genomic characterization of oesophageal carcinoma. Nature 2017;541: 169-175.

18 Evans SC, Gillis A, Geldenhuys L, et al: Microsatellite instability in eAdenocarcinoma. Cancer Lett 2004;212:241-251.

19 Kulke MH, Thakore KS, Thomas G, et al: Microsatellite instability and hMLH1/hMSH2 expression in Barrett esophagus-associated adenocarcinoma. Cancer 2001;91:1451-1457.

20 Shiraishi H, Mikami T, Aida J, et al: Telomere shortening in Barrett's mucosa and esophageal adenocarcinoma and its association with loss of heterozygosity. Scand J Gastroenterol 2009;44:538-544

21 Cai JC, Liu D, Liu KH, et al: Microsatellite alterations in phenotypically normal esophageal squamous epithelium and metaplasiadysplasia-adenocarcinoma sequence. World J Gastroenterol 2008;14:4070-4076.

22 Lin L, Wang Z, Prescott MS, et al: Multiple forms of genetic instability within a $2-\mathrm{Mb}$ chromosomal segment of 3q26.3-Q27 are associated with development of esophageal adenocarcinoma. Genes Chromosomes Cancer 2006;45:319-331.

23 Yanagi M, Keller G, Mueller J, et al: Comparison of loss of heterozygosity and microsatellite instability in adenocarcinomas of the distal esophagus and proximal stomach. Virchows Arch 2000;437:605-610.

24 Rumpel CA, Powell SM, Moskaluk CA: Mapping of genetic deletions on the long arm of chromosome 4 in human esophageal adenocarcinomas. Am J Pathol 1999;154:1329-1334.

25 Muzeau F, Flejou JF, Belghiti J, et al: Infrequent microsatellite instability in oesophageal cancers. Br J Cancer 1997;75:1336-1339.

26 Gleeson CM, Sloan JM, McGuigan JA, et al: Ubiquitous somatic alterations at microsatellite alleles occur infrequently in Barrett's-associated esophageal adenocarcinoma. Cancer Res 1996;56:259-263.

27 Meltzer SJ, Yin J, Manin B, et al: Microsatellite instability occurs frequently and in both diploid and aneuploid cell populations of Barrett's-associated esophageal adenocarcinomas. Cancer Res 1994;54:3379-3382.

28 Inamura K, Yamauchi M, Nishihara R, et al: Prognostic significance and molecular features of signet-ring cell and mucinous components in colorectal carcinoma. Ann Surg Oncol 2015;22:1226-1235.

29 Morikawa T, Shima K, Kuchiba A, et al: No Evidence for Interference of H\&E Staining in DNA Testing: Usefulness of DNA Extraction from H\&E-Stained Archival Tissue Sections. Am J Clin Pathol 2012;138:122-129.

30 Imamura Y, Lochhead P, Yamauchi M, et al: Analyses of clinicopathological, molecular, and prognostic associations of Kras codon 61 and codon 146 mutations in colorectal cancer: cohort study and literature review. Mol Cancer 2014;13:135.

31 Cancer Genome Atlas Research N: Comprehensive molecular characterization of gastric adenocarcinoma. Nature 2014;513:202-209.

32 Kim SY, Choi YY, An JY, et al: The benefit of microsatellite instability is attenuated by chemotherapy in stage II and stage III gastric cancer: results from a large cohort with subgroup analyses. Int J Cancer 2015;137:819-825. 